\title{
Damage and damping of short-glass-fibre- reinforced PBT composites under dynamic conditions: Effect of matrix behaviour
}

\author{
Dani Abdo ${ }^{\mathrm{a}, \mathrm{b}}$, Andrew Gleadall ${ }^{\mathrm{a}}$, Vadim V. Silberschmidt ${ }^{\mathrm{a},}$ \\ ${ }^{a}$ Wolfson School of Mechanical, Electrical and Manufacturing Engineering, Loughborough University, Epinal Way, Loughborough LE11 3TU, UK \\ ${ }^{\mathrm{b}}$ School of Mechanical Engineering, Rheinische Fachhochschule Koeln, Schaevenstr. 1 a-b, 50676 Cologne, Germany
}

\section{A B S T R A C T}

\begin{abstract}
The effect of a thermoplastic polyester elastomer (TPEE) on the damping properties and energy dissipation of PBT (polybutylene terephthalate) reinforced with short glass fibres is investigated in this study. Dynamic mechanical analysis (DMA) showed that tan $\delta$ increased significantly as a result of the incorporation of TPEE, especially for frequencies below $60 \mathrm{~Hz}$. With increasing frequency, the storage modulus increased and the loss moduli decreased, with the loss modulus being higher for the TPEE composite. Tensile cyclic loading tests showed that more energy was dissipated during the first loading/unloading cycle for PBT TPEE composites than for PBT ones. Damage, characterized by degradation of the elastic modulus during sequential loading-unloading cycles, was analysed at strain-rates of $0.1,0.2,0.8$ and $3.2 \mathrm{~s}^{-1}$. These experiments confirmed the DMA results and demonstrated that the TPEE composite dissipated more energy than the comparable PBT samples. SEM analysis showed that the incorporation of TPEE weakened the fibre-matrix interfacial bonding, especially in the ductile zones of the fracture surface. Moreover, localized deformation in the interface zone between glass fibres and the matrix was affected by the visco-damage behaviour, explaining the increase in the $\tan \delta$ value for the TPEE composite.
\end{abstract}

\author{
Keywords: \\ PBT \\ TPEE \\ DMA \\ Damping behaviour \\ Energy dissipation \\ SEM
}

\section{Introduction}

Polybutylene terephthalate (PBT), one of the most versatile engineering thermoplastics, is a high-performance semi-crystalline resin. Despite its outstanding mechanical and structural behaviour, it has a few limitations that need to be addressed; for example, it has low impact strength and is notch-sensitive [1]. Generally, the blending of two polymers is a simple and cost-effective way to enhance materials' mechanical properties. So, the incorporation of rubber particles into a thermoplastic polymer matrix was widely studied during the last two decades. These studies [2-5] showed that rubbery materials improve the impact properties and low-temperature toughness of the polymer. The literature contains numerous works demonstrating the effect of elastomer blending with polymers (see e.g. [6-15]).

Thermoplastic elastomer is a suitable blending material for PBT, as shown in several studies [8,16-19]. Thermoplastic polyester elastomer (TPEE), a new member in the thermoplastic-elastomer (TPE) family, is suitable for blending with PBT [19]. Chemically, its hard segments are crystalline polyesters like those of PBT [20], making it compatible in blends [19]. Recent research was focused on the effect of TPEE on the impact strength of short-glass-fibre-reinforced (SGFR) PBT. For instance, Verma et al. [17] observed significant improvement in the impact toughness of PBT/TPE blends, but a decrease in tensile strength, with increasing TPE content. The effect of TPE on impact strength and properties of SGFR PBT and similar polymers is well studied $[17,19,21-25]$. Moreover, mechanical properties of TPEE could be enhanced before incorporation with other thermoplastics by using, for example, composite particle technology [26].

Generally, the overall properties of a new composite depend on the properties of the blending materials. Adding low-modulus rubber thermoplastic to engineering thermoplastics lowers their stiffness and strength. To overcome this deficiency, high-modulus fibres can be incorporated to the matrix material. By using short glass fibres, materials can still be injection moulded into engineering products by applying the same injection process as for unreinforced thermoplastic materials [27]. While the effect of rubbery material on thermoplastics is well studied, as mentioned before, the combination of glass fibre reinforcement and rubber in engineering thermoplastics is relatively unexplored. Though PBT has many applications, it is mostly used with glass-fibre reinforcement when applied as engineering thermoplastic industrially. Different applications of PBT reinforced composites necessitate the exploration of the effect of TPEE on short-glass-fibre reinforced PBT.

\footnotetext{
* Corresponding author.

E-mail addresses: d.abdo@lboro.ac.uk (D. Abdo), a.gleadall@lboro.ac.uk (A. Gleadall), v.silberschmidt@lboro.ac.uk (V.V. Silberschmidt).
} 
For instance, the interaction and synergetic effects of TPEE on the interfacial matrix-fibre bonding is of high interest. The effect of TPEE on dynamic behaviour, including damping and energy dissipation as well as the effect of matrix on its interfacial bonding with glass fibres, is so far unstudied.

The aim of this paper is first to study the effect of TPEE on damping properties of short-fibre-reinforced PBT composites. Results of incremental tensile tests carried out at quasi-static and dynamic conditions are analysed, and energy dissipation is compared for both materials. Damage initiation and evolution at different stress levels is studied macroscopically. Second, the study aims to identify the mechanism of deformation and damage occurring at the microscopic scale using SEM observations. The effect of viscous behaviour of the PBT matrix on the interfacial damage is analysed and linked to the overall performance of both studied composites.

\section{Experimental setup and methodology}

\subsection{Materials and specimens}

In this study, two PBT composites are investigated. The first, standard polybutylene terephthalate with $10 \mathrm{wt} \%$ glass-fibre content referred to as PBT-GF10 is used as a reference material to evaluate the effect of TPEE on fibre-reinforced PBT composites. The second material, polybutylene terephthalate with $10 \mathrm{wt} \%$ glass fibres and $10 \mathrm{vol} \%$ thermoplastic polyester elastomer (TPEE), is referred to as PBT-GF10 TPEE. All samples used in experiments were standard ISO 527 dogbone specimens produced via injection moulding.

\subsection{Dynamic mechanical analysis}

Dynamic mechanical analysis (DMA) of the composites was performed using a Mettler Toledo DMA machine at room temperature. Frequency sweeps were performed for a range between $0.1 \mathrm{~Hz}$ and $100 \mathrm{~Hz}$; temperature and strain were kept constant. The force offset and force amplitude were chosen after performing force sweeps. These preliminary experiments were important to ensure running the experiment at the highest possible value of modulus. The analysis was done on a 3-point-bending clamping system, which is most appropriate for fibre-reinforced polymers. The specimen's dimensions were $90 \mathrm{~mm}$ (length), $10 \mathrm{~mm}$ (width) and $4 \mathrm{~mm}$ (thickness).

\subsection{Hysteresis loss}

A hysteresis loss is the amount of energy dissipated during cyclic (loading/unloading) deformation. Samples are stretched at a specific strain rate to a chosen stress level below the stress value at fracture and then allowed to retract to initial unstretched state at the same strain rate. The hysteresis loss was determined for tensile dogbone specimens for both studied materials using a Zwick Z010 machine equipped with a $10 \mathrm{kN}$ load cell. All the tests were performed using an extensometer with a $10 \mathrm{~mm}$ gauge length. The tests were carried out at strain-rates of $0.1,0.2,0.8$ and $3.2 \mathrm{~s}^{-1}$.

\subsection{SEM observations and morphological characterisation}

Morphological analysis was completed using a Phenom XL scanning electron microscope with a range of accelerating voltage from 5 to $15 \mathrm{kV}$. To prevent specimens from charging, a charge-reduction mode was activated. This mode produced best results in terms of noise reduction and quality of micrographs. An additional SEM system (JSM$7500 \mathrm{~F}$ ) with the same range of accelerating voltage was also used. For investigations with this microscope, specimens were sputter-coated with a $10-15 \mathrm{~nm}$ thick layer of gold-palladium to provide an efficient charge transfer. The aim of employing two different microscopes and methods was to ensure that there were no losses in image quality due to the charging problem. Gold-palladium coating was effective to obtain high-quality images of the matrix but had a negative effect on observation of matrix residue on pulled-out fibres.

\section{Results and discussion}

\subsection{Distribution and orientation of glass fibres}

The mechanical properties of SGFR thermoplastic composites are strongly affected by the size and orientation of glass fibres in the final reinforced thermoplastic composite [28]. Shear forces, immanent in the process of injection moulding, lead to significant reduction in fibre length, which, in turn, may cause decrease in ultimate strength [29]. Thus, while comparing SGF reinforced thermoplastic composites, it should be taken into consideration that different length, orientation and distribution of the fibres, which are hard to influence during the injection process, can lead to a scatter in results, especially for tests at very low strain rates, as in DMA analysis. The distribution and orientation of glass fibres can differ from each mould to another and depend on properties of the polymer matrix and its viscosity during the injection moulding process. In this study, the orientation and distribution of fibres in the thermoplastic matrix was investigated by image analysis on SEM micrographs.

The micrographs of longitudinal and transverse cross-sections of specimens of both materials show that most fibres are not oriented randomly but relatively aligned along the mould flow direction (Fig. 1(a,c)); this could be observed by cross-sections of the fibres noticed in longitudinal cross-sections. However, these fibres are not optimally oriented along the same direction vector as seen in the transverse cross-sections of both materials (Fig. 1(b,d)). This could be concluded based on the divergency of directions of single fibres and the differences in their lengths observed in the transverse cross-sections. Moreover, it can be observed that the fibre distribution varies across the view plane, and therefore, the matrix of both materials is non-uniform. As a conclusion, the orientation of fibres which affects greatly the strength and stiffness of the reinforced material, was similar in both materials and there was no significant effect of TPEE on the alignment of these fibres.

\subsection{Viscoelastic properties of SGF PBT composites: frequency sweep measurements}

Rheological testing of thermoplastic materials can be performed on both solid samples and melts. Product-performance issues are usually related to properties of solid samples [30]. Rheological properties of the studied materials were investigated in the region of linear viscoelasticity. In this study, the viscosity of solid samples as a function of angular frequency at room temperature was obtained from DMA experiments and presented in Fig. 2.

\subsubsection{Complex viscosity of SFR PBT composites}

It could be observed that both PBT composites showed Newtonian behaviour in the frequency dependence within the investigated range of shear rates. As can be seen, TPEE/PBT composite has a higher viscosity at room temperature than the PBT one. It was reported [31] that glass fibres could affect the level of viscosity of composite materials by hindering the movement of a matrix skeleton and chain segments. As the dimensions and volume fractions of glass fibres are similar in both materials, the increased value of complex viscosity could be attributed only to the addition of TPEE and not to any effect of glass fibres. PBTGF10 TPEE had a higher viscosity over the whole frequency range. At lower frequencies the value of viscosity of PBT-GF10 TPEE was $90 \%$ larger than that of the reference material. With increasing frequency these values converged, and the difference decreased to $15 \%$ at the end of the used frequency range. 

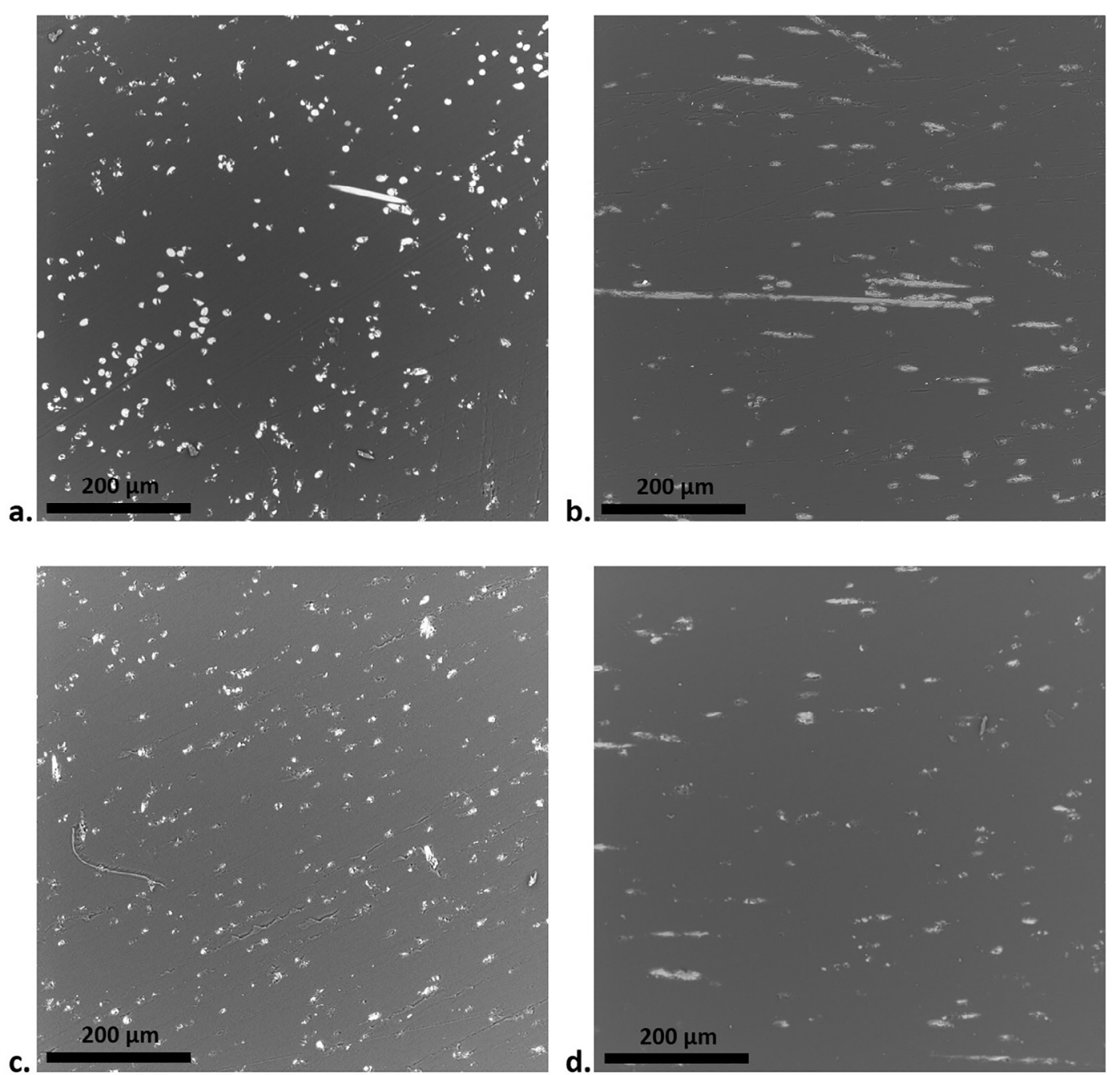

Fig. 1. SEM observations of fibres' distribution and orientation in longitudinal (a and c) and transversal (b and d) cross-sections: (a and b) PBT-GF10; (c and d) PBTGF10 TPEE.

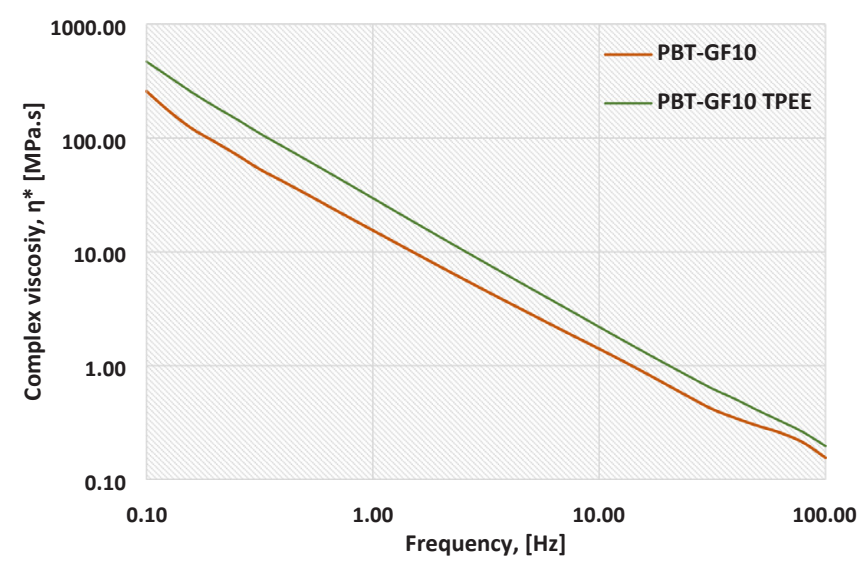

Fig. 2. Complex viscosity versus frequency for PBT composites with and without TPEE at $23^{\circ} \mathrm{C}$.

\subsubsection{Storage modulus $E^{\prime}$ of $P B T$ composites}

The variation of the storage modulus E' for PBT-GF10 TPEE and the reference material PBT-GF10 is depicted in Fig. 3. The value of this modulus is linked to the material's ability to store energy elastically. It is apparent that the magnitude of the storage modulus $E^{\prime}$ for both materials increases with increasing frequency; the trends for both materials were generally similar. The difference in the value of the

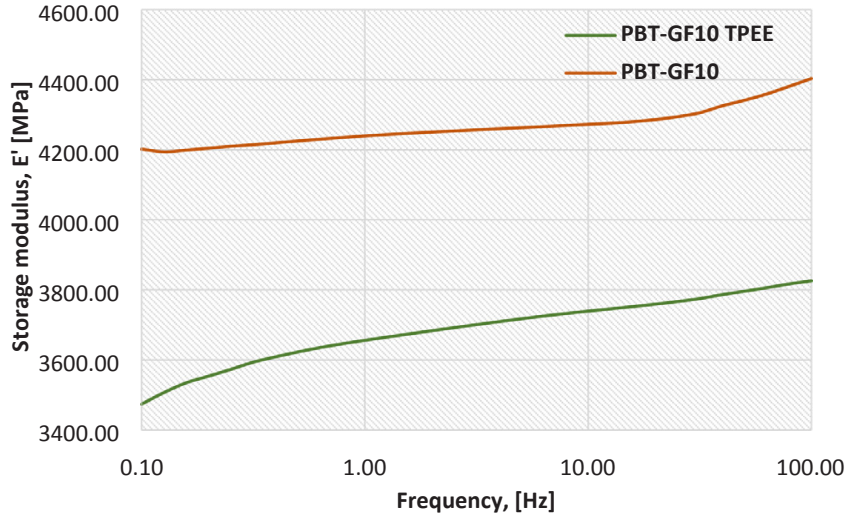

Fig. 3. Variation of storage modulus of PBT-GF10 and PBT-GF10 TPEE with frequency.

modulus between them could be attributed to the incorporation of TPEE which as reported by some researchers [17]. It can be noticed that there was an acceleration of increase in storage modulus for PBT-GF10 after $50 \mathrm{~Hz}$ (short relaxation times), whereas, for the TPEE material, a high increase in the value of E' was noticed between $0.1 \mathrm{~Hz}$ and $1 \mathrm{~Hz}$ (long relaxation times). This could be referred to a change in viscous behaviour of the material in this range of frequency, which, in turn 


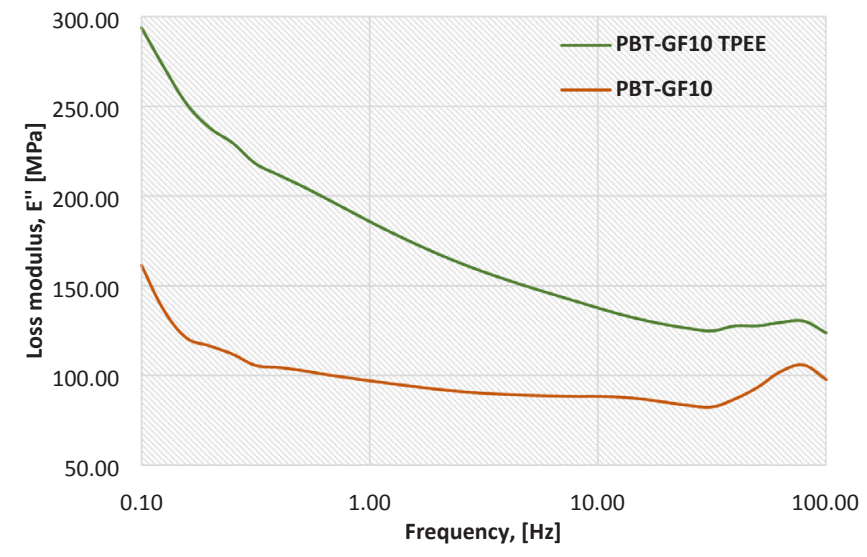

Fig. 4. Variation of loss modulus of PBT-GF10 and PBT-GF10 TPEE with frequency.

could affect the bonding forces between fibres and the matrix, leading to a change in the elastic-modulus values. Basically, increasing the frequency means a decrease in the time scale of the stress input. In this case, the specimen has less time to relax, with its response becoming more elastic, as can be observed in the monotonic increase of $\mathrm{E}^{\prime}$.

\subsubsection{Loss modulus $E^{\prime \prime}$ of PBT composites}

The loss modulus $E^{\prime \prime}$ of the studied composites demonstrated a different trend: both materials showed decreasing $E^{\prime \prime}$ as frequency increased. The loss modulus E" characterises the viscous behaviour of the material and its ability to dissipate stress through heat, signifying a correlation of the stress to a dissipated energy. The effect of TPEE on energy-dissipation ability of PBT composites can be clearly observed in Fig. 4. A significant increase in $E^{\prime \prime}$ could be noticed in a low-frequency range. At $0.1 \mathrm{~Hz}$, a $100 \%$ increase in $\mathrm{E}^{\prime \prime}$ value due to TPEE addition: from $150 \mathrm{MPa}$ to approximately $300 \mathrm{MPa}$. The value of E" for PBT-GF10 exhibited a maximum in the low-frequency range. This value decreased with frequency increasing between $0.1 \mathrm{~Hz}$ and $5 \mathrm{~Hz}$, and slightly declined to a minimum at $50 \mathrm{~Hz}$ before displaying an increase. On the other hand, the E" value of the TPEE material had a maximum value of $300 \mathrm{MPa}$ at $0.1 \mathrm{~Hz}$, which decreased gradually with increasing frequency. In a higher-frequency range - between $50 \mathrm{~Hz}$ and $100 \mathrm{~Hz}$ - the value reached a plateau of around $135 \mathrm{MPa}$. The plots of the two curves show a convergent trend at the end of the employed frequency range, demonstrating that TPEE had a significant effect on energy dissipation, especially for frequencies below $60 \mathrm{~Hz}$. The trends for E" for both materials agreed with those observed for $\mathrm{E}^{\prime}$ and the variation of viscosity as function of angular frequency. In general, as the elastic contribution of a specific material $\mathrm{E}^{\prime}$ increases, the system becomes less viscous, as could be seen in Fig. 2. With decreasing viscosity less energy could be dissipated, i.e. $\mathrm{E}^{\prime \prime}$ decreases with increasing frequency. The movement of molecular chains is restricted, and the interfacial dissipation behaviour decreases.

\subsection{4. $\tan \delta$ of PBT composites}

Tan delta $(\delta)$ represents the ratio of a viscous response to an elastic one of a viscoelastic material. In other words, $\tan \delta$ stand for the energy dissipation and indicates the effectiveness of a material's damping capability. It expresses the out-of-phase time relationship between an impact force and the transmitted resultant force.

The values of $\tan \delta$ with respect to frequency for the studied SGFR composites are presented in Fig. 5. It can be noticed that the $\tan \delta$ values for PBT-GF10 TPEE - compared to those of PBT-GF10 - were higher in the entire frequency range. The difference in $\tan \delta$ values observed for both PBT composites indicate different amounts of energy absorbed by each material. Generally, when a composite is subjected to external stress, the energy can be dissipated by friction at fibre-fibre,

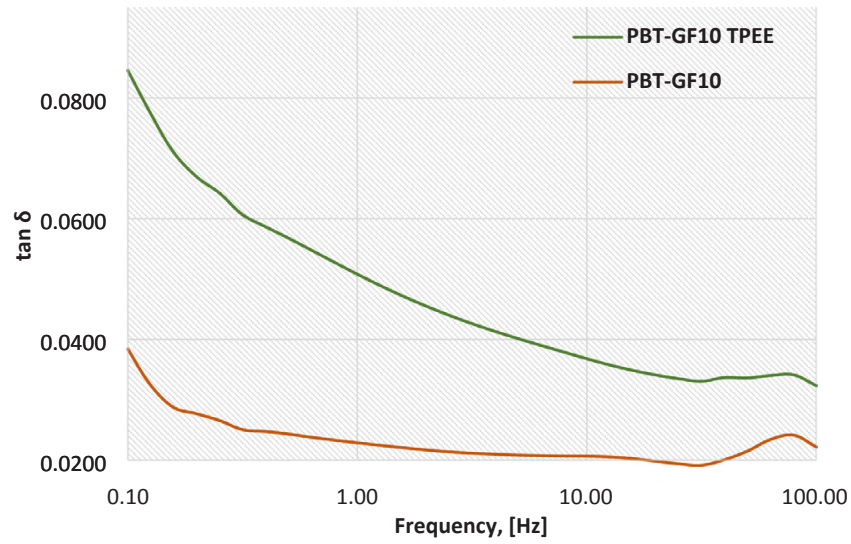

Fig. 5. Variation of $\tan \delta$ of PBT-GF10 and PBT-GF10 TPEE with frequency.

polymer-polymer and fibre-polymer interfaces.

At frequencies below $1 \mathrm{~Hz}$, the $\tan \delta$ value of the TPEE composite was 2.2-2.9 times higher than that of PBT-GF10. With increasing frequency $\tan \delta$ decreased rapidly to reach a plateau between $50 \mathrm{~Hz}$ and $100 \mathrm{~Hz}$. On the other hand, the $\tan \delta$ value for PBT-GF10 decreased at low frequency and had almost consistent slight decrease at frequency increasing frequency between $0.5 \mathrm{~Hz}$ and $50 \mathrm{~Hz}$, where a minimum in observed followed by a slight increase between $60 \mathrm{~Hz}$ and $90 \mathrm{~Hz}$.

The higher $\tan \delta$ value for the PBT-GF10 TPEE material could be related to higher energy absorbed through viscous flow of the rubber matrix (TPEE) and interfacial damping between the PBT and rubber as well as the energy dissipated by micro-movements of glass fibres due to the difference in structural behaviour of the matrix. With increasing frequency, the ability of polymer-chain movements is restricted, which explains the decrease in the loss modulus of both materials.

The performed DMA analysis showed the effect of TPEE on rheological behaviour of SFR PBT composites, with an increase in viscosity value observed for the TPEE material, in addition to an increase in the loss modulus E". This means that more energy can be dissipated through heat. These observed effects led to enhancement of TPEE material's damping capability represented by $\tan \delta$.

\subsection{Hysteresis}

Viscoelastic materials exhibit specific features, different from elastic materials. Most notably, ideal elastic materials store $100 \%$ of their energy as recovery elastic strain energy when deformed. In contrast, viscoelastic materials lose, or dissipate a portion of this energy; this dissipation is known as hysteresis. Explicitly, a hysteresis requires that data points of the loading portion of the stress-strain curve have higher stress values than those of the unloading curve.

The hysteresis loss is thus the area between the loading and unloading curve of the stress-strain diagram represented and calculated as

Hysteresis loss $=$ Area under loading curve - Area under recovery curve.

In this section, a response of the PBT composites to two kinds of cyclic loading is studied:

1. Cyclic loading with constant stress values,

2. Cyclic loading with increasing loading increments.

The test results should help to analyse the energy dissipated for individual cycles and calculate the damage induced for different stress levels before and after yielding of the material.

\subsubsection{Damage evolution in PBT composites}

Cyclic tests were performed for loads corresponding to $70 \%$ of 

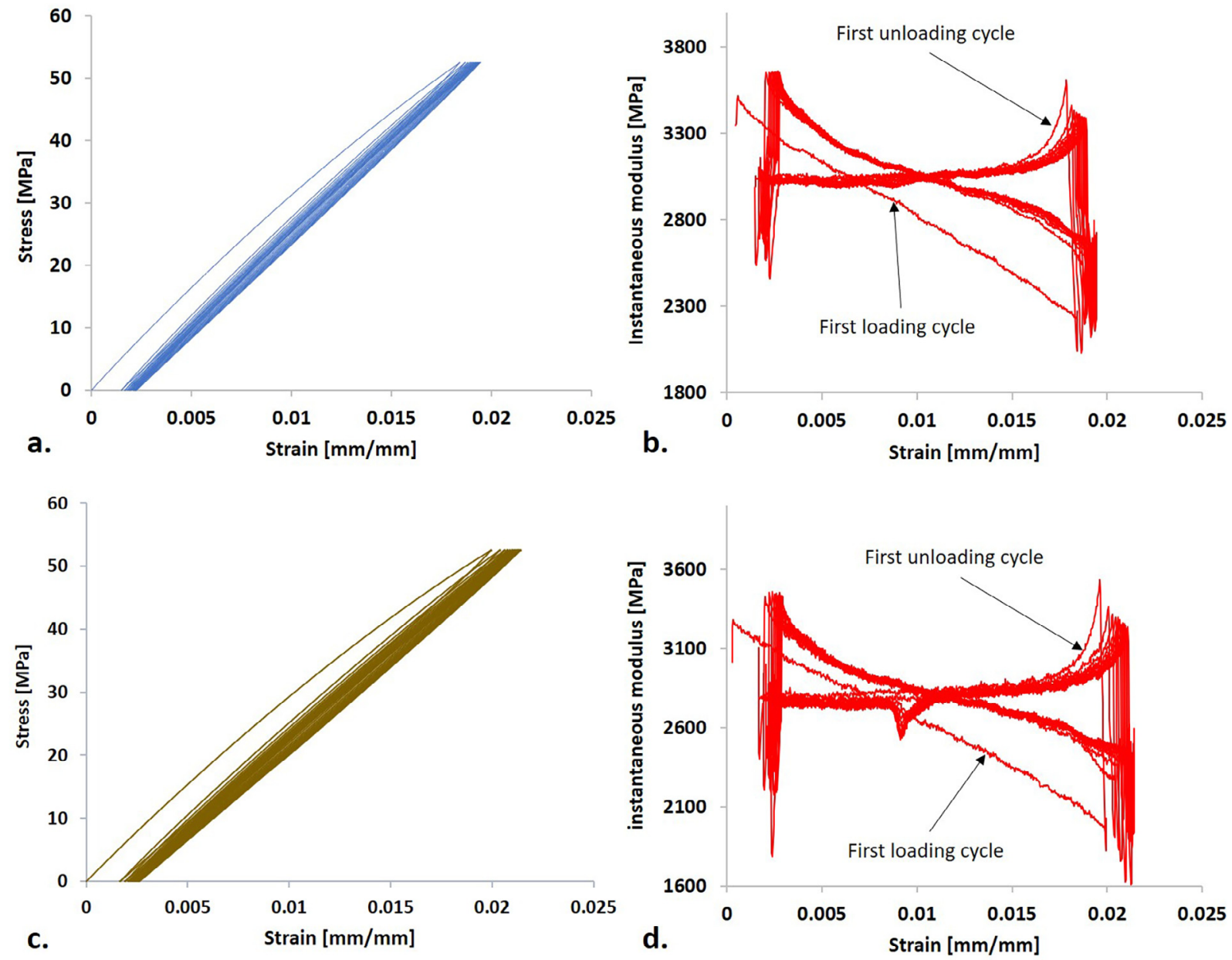

Fig. 6. (a) cyclic loading of PBT-GF10; (b) instantaneous modulus of PBT-GF10; (c) cyclic loading of PBT-GF10 TPEE; (d) instantaneous modulus of PBT-GF10 TPEE.

fracture stress obtained from uniaxial tensile testing $(2100 \mathrm{~N}$ load at $2 \mathrm{~mm} / \mathrm{min}$ ). The energy dissipated during repetitive cycles for PBTGF10 and PBT-GF10 TPEE was analysed, and the amount of deformation and damage induced within each cycle was quantified. For each specimen, 13 cycles were completed.

The monotonic cyclic stress-strain curves and their related instantaneous modulus plotted are given in Fig. 6. Apparently, the most damage and plastic deformation occurred within the first cycle. The residual plastic strain measured after each cycle was higher for the material with TPEE at all cycles, indicating its higher plasticity. This could be due to debonding or fracture of glass fibres; obviously for a specific stress level, a definite amount of debonding would occur. This is mostly due to the material's heterogeneity and the non-uniform distribution and orientation of fibres. Fibres with weaker bonding or greatest misorientation from the optimal loading direction would be first to debond. Then, damage progressively propagates to less misoriented fibres in the subsequent cycle. For the load, increasing with each cycle, more debonding should happen at each stress level until the stress level capable of separating or breaking enough fibres allowing microcracks to grow and accumulate would lead to total fracture. For cycles with a constant stress level, the dissipated energy, damage and plastic deformation converge to minimum nearly constant values. At this level, most potential damage (corresponding to this stress level) has already occurred. The plots of the instantaneous modulus of both materials clearly demonstrate its reduction due to damage induced during the tensile pulling. This reduction could be observed by the change of position of the curves on the x-axis of the diagram. The values of Young's modulus for individual cycles listed in Table 1 show that the
Table 1

Values of dissipated energy, plastic strain and Young's modulus for PBT composites.

\begin{tabular}{lllllll}
\hline \multirow{2}{*}{$\begin{array}{l}\text { Cycle } \\
\end{array}$} & $\begin{array}{l}\text { lissipated Energy [MJ/ } \\
\left.\mathrm{m}^{2}\right]\end{array}$ & $\begin{array}{l}\text { Residual Strain } \\
{[\%] * 10^{-3}}\end{array}$ & \multicolumn{2}{l}{ Young's Modulus [MPa] } \\
\cline { 2 - 6 } & PBT-GF10 & PBT/TPEE & PBT-GF10 & PBT/TPEE & $\begin{array}{l}\text { PBT- } \\
\text { GF10 }\end{array}$ & $\begin{array}{l}\text { PBT/ } \\
\text { TPEE }\end{array}$ \\
\hline 1 & 79007 & 91836 & 0 & 0 & 3050 & 2800 \\
2 & 30446 & 37445 & 148 & 160 & 3035 & 2775 \\
3 & 27215 & 33405 & 166 & 191 & 3035 & 2775 \\
4 & 25974 & 31554 & 178 & 206 & 3035 & 2760 \\
5 & 25301 & 30967 & 187 & 217 & 3035 & 2750 \\
6 & 24943 & 30608 & 194 & 225 & 3030 & 2750 \\
7 & 24559 & 30399 & 199 & 232 & 3030 & 2750 \\
8 & 24459 & 30132 & 205 & 239 & 3025 & 2750 \\
9 & 24344 & 30091 & 209 & 245 & 3020 & 2750 \\
10 & 24317 & 30004 & 214 & 250 & 3015 & 2740 \\
11 & 24301 & 29962 & 218 & 254 & 3015 & 2740 \\
12 & & & 222 & 258 & 3010 & 2710 \\
13 & & & 226 & 263 & & \\
\hline
\end{tabular}

greatest reduction in the modulus happened after the first loop for both materials. The rest of the cycles caused minimal gradual degradation in the modulus since less damage happened in the specimen Table 1.

The plastic deformation of the material could be evaluated in terms of the residual strain, measured after each cycle (Table 1). Incremental values of plastic strain for each individual cycle are illustrated in Fig. 7(a), where the difference in plastic strain between each two consequent cycles is compared for both materials. It can be concluded 

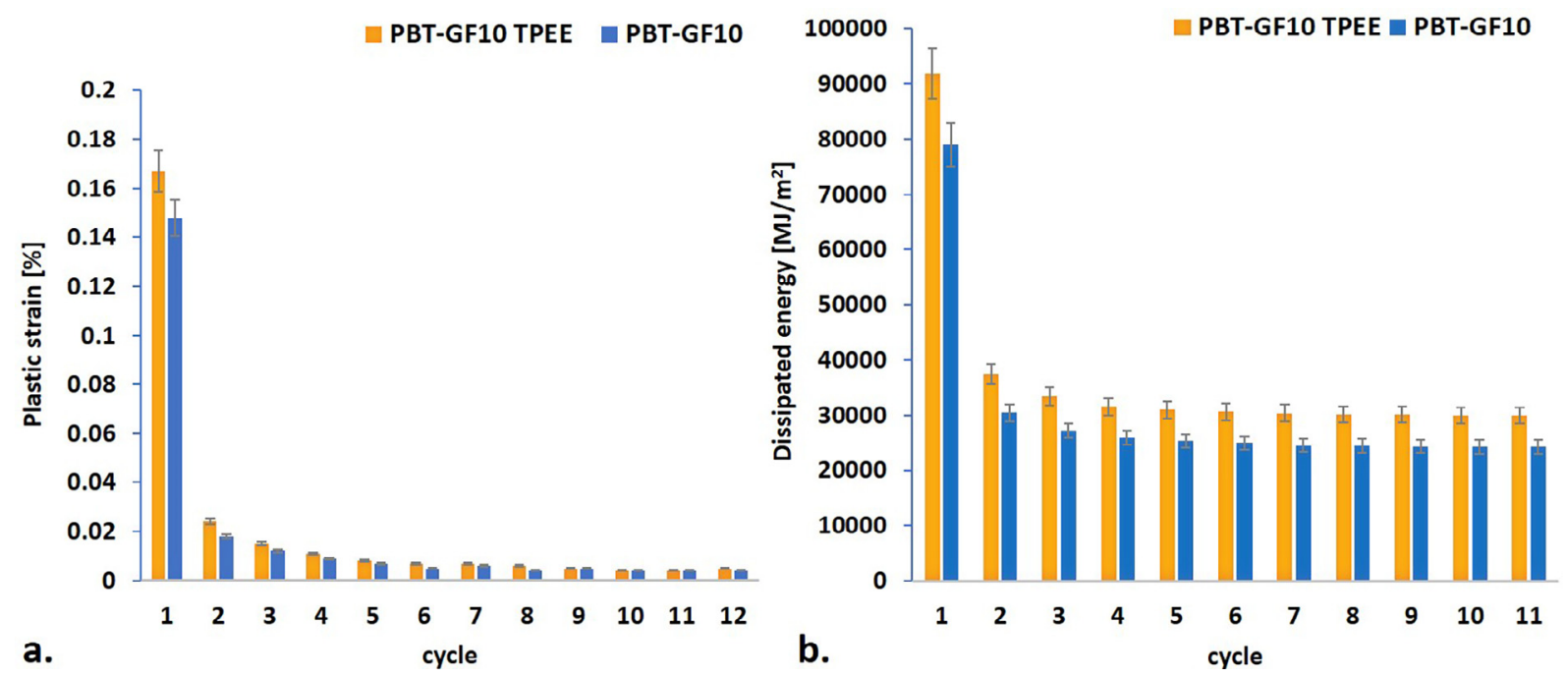

Fig. 7. Comparison of plastic strain (a) and dissipated energy (b) per cycle for PBT-GF10 and PBT-GF10 TPEE.

that the plastic deformation in the first cycle was much higher than that in the rest of the cycles. The plastic strain per cycle settled, decreased significantly, after three or four cycles; this was found for both materials. In addition, the plastic deformation measured for PBT-GF10 TPEE was higher for all cycles.

When comparing the hysteresis loss of both materials, it is observed that the amount of energy dissipated in the first cycle greatly exceeded that in the following cycles. The dissipated energy for PBT-GF10 TPEE material was higher for PBT-GF10 TPEE at all cycles as can be seen in Fig. 7(b). These findings confirmed the DMA results obtained. The dissipated energy dropped considerably after the first cycle, when the most plastic deformation and debonding occurred. The decrease during the second and the third cycle is significantly smaller but damage still happened. After the fourth cycle, the energy level reached a plateau. This value should represent the viscous energy dissipated in the damaged material as the plastic increment converges to 0 . It can be noticed that the presence of TPEE in the PBT composite led to increase in this value by $8 \%$.

\subsubsection{Hysteresis loss for uniaxial loading with variable loading increments}

The hysteresis loops were observed in the cycles with increasing loading increments $-300 \mathrm{~N}$ in each cycle - to fracture for four strain rates. This allowed the energy dissipated before and after yielding of the material for different stress levels and strain rates to be studied. The

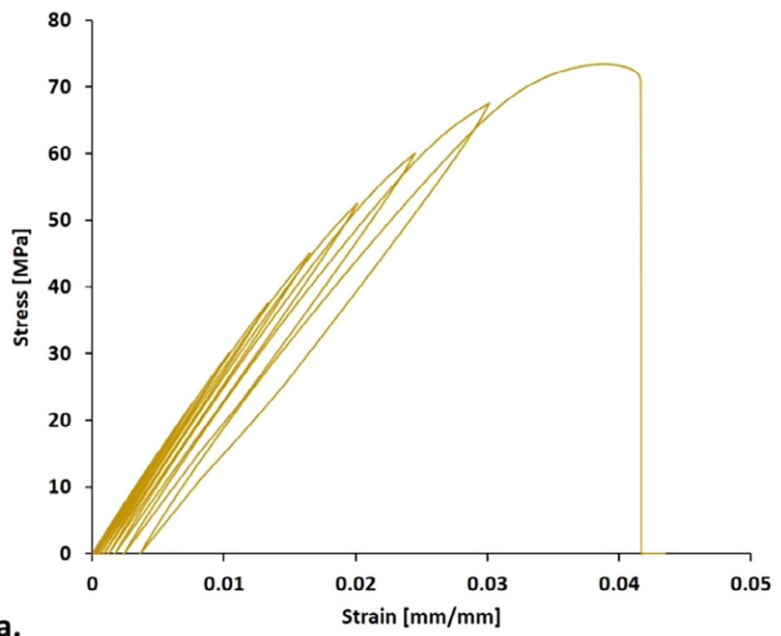

a.

Fig. 8. Quasi-static incremental loading stress-strain curves: incremental stress-strain curves, including all loops, for PBT-GF10 and PBT-GF10 TPEE are presented in Fig. 8.

The uniaxial tensile testing with loading increments was performed for both materials at strain rates of $0.1,0.2,0.8,3.2 \mathrm{~s}^{-1}$ to assess dynamic performance of these composites.

A comparison of the hysteresis loss of both composites at different strain rates is presented Fig. 9. Three major observations could be derived:

(i) The increase in the load amplitude resulted in an accelerated growth of energy, dissipated in each cycle.

(ii) The hysteresis loss for the PBT-GF10 TPEE material was higher in each individual cycle than that of PBT-GF10 at all strain-rates.

(iii) The energy dissipation decreased with increasing strain-rate, demonstrating the time-dependent viscoelastic behaviour of both materials. These conclusions confirm the observations obtained with DMA, where $\tan \delta$ for PBT-GF10 TPEE was higher than that of PBT-GF10, and values of both $\tan \delta$ and loss modulus decreased with increasing frequency (high strain-rates).

\subsubsection{Strain-rate effect on damage at macroscopic scale}

To assess the rate sensitivity of damage in terms of its initiation and growth and to evaluate the effect of TPEE on damage behaviour, the damage analysis was conducted based on the obtained experimental

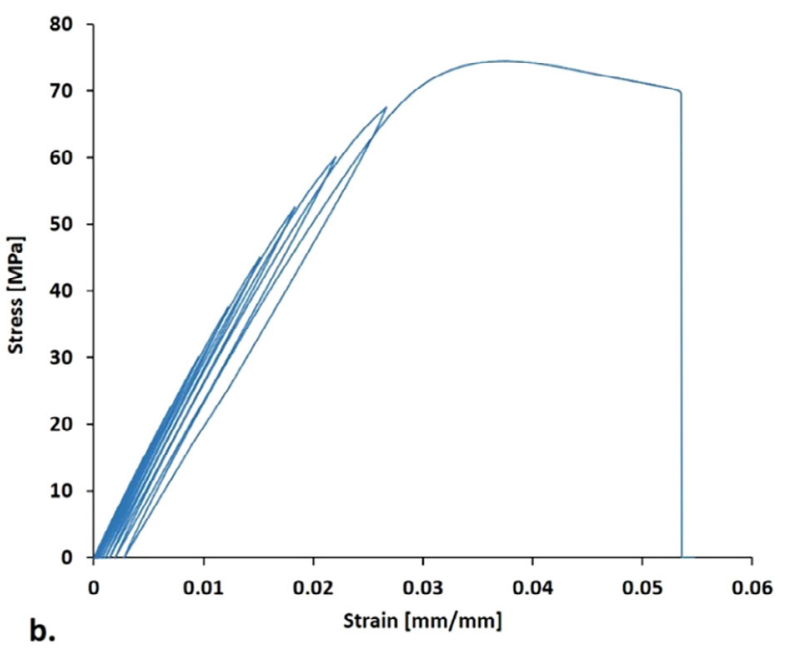

b.

(a) PBT-GF10 TPEE; (b) PBT-GF10 (strain-rate $0.2 \mathrm{~s}^{-1}$ ). 

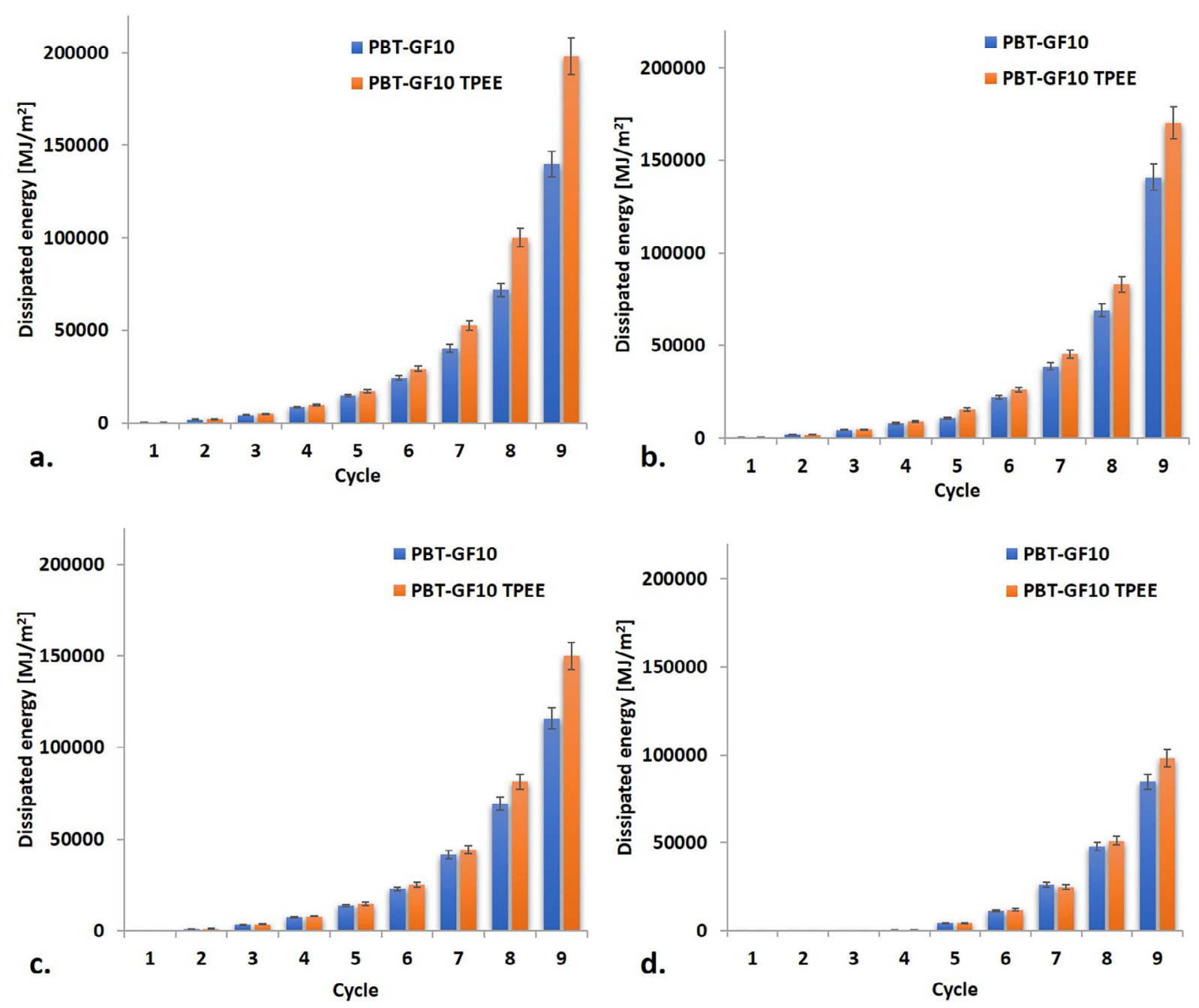

Fig. 9. Dissipated energy per cycle for cycles with increasing increments of $300 \mathrm{~N}$ for different strain rates: (a) $0.1 \mathrm{~s}^{-1}$; (b) $0.2 \mathrm{~s}^{-1}$; (c) $0.8 \mathrm{~s}^{-1}$; (d) $3.2 \mathrm{~s}^{-1}$.

results. Damage can be quantified degradation of the tensile modulus using continuum damage mechanics. The damage induced was calculated for each individual cycle for both quasi-static and dynamic loading regimes and plotted as function of strain to analyse the damage evolution for the whole experiment. The damage variable (D) is thus expressed:

$D=1-\frac{E_{D}}{E_{0}}$,

where $E_{0}$ is the modulus of the material without damage. $E_{D}$ is the residual modulus of the damaged material estimated from the slope of the unloading curves.

The damage was calculated from incremental loading stress-strain curves carried out for two strain-rates: $0.1 \mathrm{~s}^{-1}$ and $3.2 \mathrm{~s}^{-1}$, representing quasi-static and dynamic conditions. The evolution of the damage was estimated and plotted as function of strain values measured at the end of each loading increment for both materials and strain rates as shown in Fig. 10. Experimental results demonstrate a delay in damage initiation with increasing strain rate for PBT-GF10 and PBT-GF10 TPEE. It can be observed that for PBT-GF10 and PBT-GF10TPEE the first measurable damage appears at strain between 0.002 and 0.003 for quasistatic loading. However, under dynamic conditions the first measurable damage appears at strain of $0.49 \%$ and $0.97 \%$ for PBT-GF10TP and PBT-GF10, respectively. It can be thus concluded that the stiffness reduction in these composites is rate-dependent. Moreover, the damage level reached for PBT-GF10 TPEE was higher than that observed for
PBT-GF10 for both strain rates, reaching a max of 0.24 for PBT-GF10 TPEE in quasi-static loading and 0.23 in dynamic one whereas the respective values for PBT-GF10 were only 0.2 and 0.17 . To conclude, the damage for PBT-GF10 TPEE occurred at lower strain values compared to that for PBT-GF10. A higher damage level percentage was observed at each cycle for the TPEE composite, showing that this material underwent damage at low stress levels; this explains the high damping behaviour observed in DMA and the vales of hysteresis loss calculated in the previous section.

\subsection{Microscopic analysis}

This section aims to establish a link between the observations obtained at the macroscopic level in the experimental studies and microdefects, observed on fracture surfaces of both materials. This will help to emphasize the effect of TPEE on the matrix microstructure and its effect on the damping and fracture behaviour of PBT composites.

The fracture surfaces obtained in tensile tests were examined with SEM (see a typical fractogram in Fig. 11). The higher damping coefficient measured for the TPEE composite in the during DMA analysis and the larger dissipated energy observed in the incremental loading and cyclic tests under constant load could result from friction and movement of polymer chains against each other or against the glass fibres. Moreover, several studies showed that short-fibre-reinforced composite materials fail as a result of fibre-matrix interface debonding, fibre breakage and matrix micro-cracking [32]. It is unlikely that the glass 

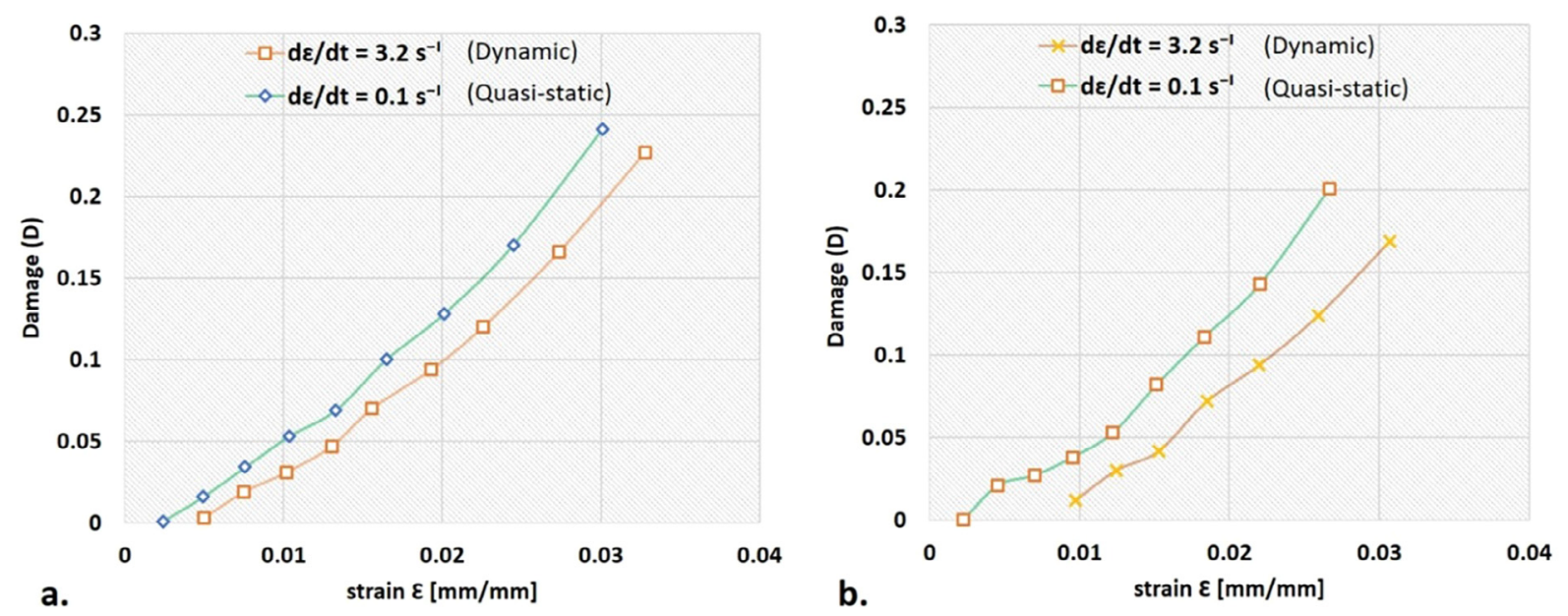

Fig. 10. Damage for quasi-static and dynamic cases: (a) PBT-GF10 TPEE; (b) PBT-GF10.

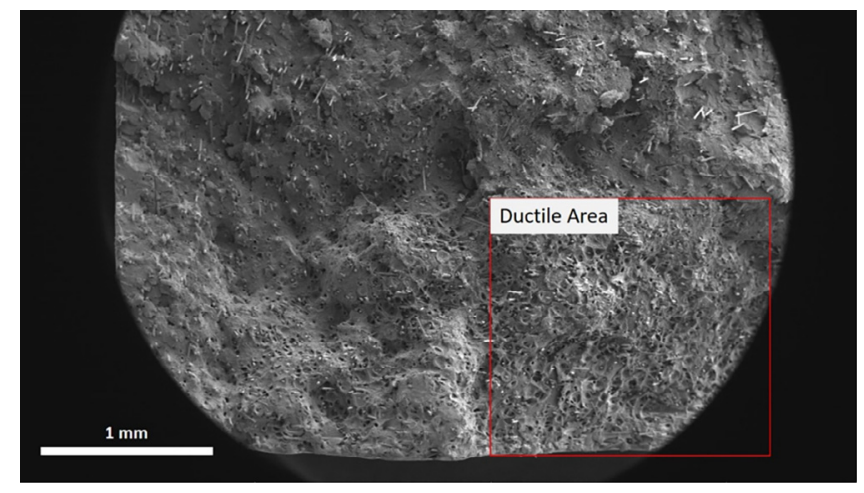

Fig. 11. Ductile area with different microstructure from brittle fracture region: Fracture surface of PBT-GF10 TPEE.

fibres elongated to a significant degree because of their high elastic modulus relative to that of the matrix. It is more likely that deformation of the material occurred around the fibres.

The fracture surfaces of both material composites are presented in Fig. 12. The fracture surfaces showed a region covered with pulled-out fibres and stretched dimples of the matrix, which is a characteristic ductile-failure mode of the matrix (Fig. 11). The area of ductile behaviour was measured for specimens tested at different strain-rates, and it was found that its size decreased with increasing strain rate. Moreover, bigger ductile areas were observed for the TPEE material at all the used strain rates. The rest of the fracture surface surrounding the ductile area showed a clear brittle failure mode, characterised by its relatively flat surface. It is considered that the ductile area is the region of crack initiation, with the crack propagating through the specimen in a brittle manner, leading to final failure of the specimen [33]. However, despite the similarities in fracture mode, ductile areas of the two composites show clear differences. It was observed that more fibres were pulled out of the matrix in the ductile areas of PBT-GF10 TPEE when compared to PBT-GF10 indicating weakening of fibre-matrix adhesive bonding (see arrows in Fig. 12(a,c)). In the brittle area, matrix residue could be found on the pulled-out fibres. It can be seen that for PBT-GF10 the fibres were completely covered with this matrix residue (Fig. 12(d)). It could be clearly observed that the TPEE-blended material had a relatively weaker fibre-matrix adhesion - similar observation was reported for GF reinforced TPEE and was referred to decreased crystallinity [34] - in the brittle area, as concluded from the amount of matrix residue on the fibres (Fig. 12(b)). This can explain the higher levels of stiffness and tensile strength of the PBT-GF10 material. The fibres prevent polymer chains from moving when the material is exposed to external stress.
Higher interfacial bonding led to higher stiffness and rigidity, hence having negative effects on the damping behaviour.

In the ductile areas, higher plastic deformation could be observed in the matrix of TPEE material. Voids formed around pulled-out and existing fibres, as can be seen in Fig. 12(a). This behaviour is significantly less pronounced in PBT-GF10. The stretching of the matrix during loading could accelerate the debonding rate. Analysis of cryogenically fractured specimens showed no voids or stretching for loaded or unloaded specimens, indicating that voids were formed during loading. As damage was detected at lower stress levels (see in the previous section), it should be mentioned that closure of micro-cracks could occur during the unloading phase especially at low stress strain levels. However, microcracks do not close completely. During cyclic loading, the inelastic (permanent) strain measured after unloading was caused by the subsequent crack opening explaining the damage accumulation and could lead to higher damage found in the TPEE material. Significantly higher local deformation happened in the PBT/TPEE matrix, especially in the ductile areas and around the fibres on a larger scale (consider the ductile-area size). When the load was released, the structure underwent some kind of healing process, which could be seen on the tensile curves of both materials (Fig. 6(d)). This high deformation potential can explain the higher values of $\tan \delta$ observed in the DMA analysis. As viscoelasticity is time-dependent these behaviours were suppressed with increasing levels of frequency or strain rates. Since damage or ductile areas need time to form, higher strain rates resulted in their lower levels.

The deformation of the matrix due to fibre-pulling could be observed for both materials in Fig. 13. The TPEE composite shows, as mentioned before, higher deformation in the ductile area. This kind of deformation, characterized by pulling out of the material, was not noticed in PBT-GF10 for any strain rate. Fig. 13(a) demonstrates a partially pulled-out fibre on TPEE composite fracture surface. Apparently, good adhesive behaviour could be observed on the part of the fibre in contact with the matrix (Fig. 13(a): arrow 1). However, one can also notice (Fig. 13(a): arrow 2) that the pulled-out part shows no matrix residue, an indication for a weak bonding. The observed deformation mechanism clearly shows another type of debonding in PBT-GF10 TPEE as compared to PBT-GF10. When the matrix material deformed one part of a fibre with the weaker adhesion was pulled out, while the other end with strong adhesion pulled the material out, forming a conic structure. This deformation is responsible for the composites' ductile potential, which explains the better damping properties and higher loss-modulus values, as micro-stretching or movement of the material around glass fibres compensated the stiffness increase, caused by fibre reinforcement. These conic-shaped structures formed by micro-deformations, dissipate energy, explaining the higher hysteresis loss observed 

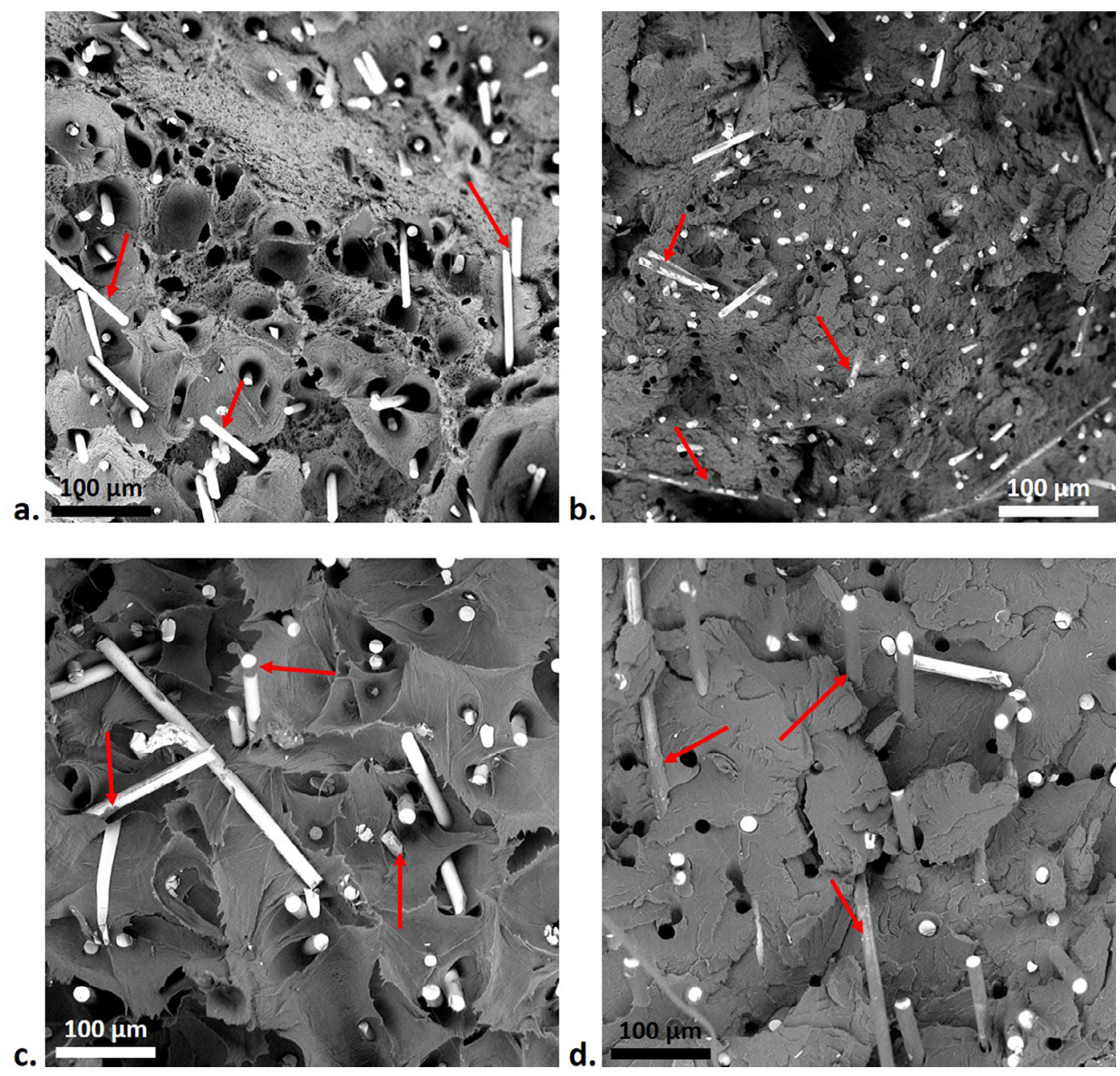

Fig. 12. Ductile (a) and brittle (b) areas of TPEE composite. Ductile (c) and brittle (d) areas of PBT-GF10.

(Fig. 13(b)).

On the other hand, in a similar position for PBT-GF10 (its ductile area (Fig. 13(c)) shows PBT-GF10, most of the fibres were broken, a clear indication of efficient high matrix-fibre bonding. Moreover, no matrix micro-cracking or significant void opening was observed around the fibres in contrast to the TPEE material. However, apparently, the matrix was strained around the glass fibres as can be seen in Fig. 13(c,d): (see arrows). This strained zone around the debonded interface dissipated the strain energy and inhibited the crack-propagation through the matrix. Similar observation was made for glass-fibre-reinforced ethylene-propylene composite at high strain rates [32].

The difference in the damage happening in the ductile areas for both materials clearly confirms the experimental results and the effect of the TPEE on the matrix behaviour of TPEE composites. Such localized deformation in the interface zone around glass fibres affected the viscodamage behaviour in presence of TPEE, acting as dissipation zones and explaining the increase in the damping coefficient $\tan \delta$.

\section{Conclusion}

Specimens of PBT-GF10 and PBT-GF10 TPEE composites were tested dynamically in DMA and under uniaxial tensile cyclic testing, their damping properties and hysteresis loss, as well as damage evolution, were compared. It was found that the addition of TPEE into glass-fibre-reinforced PBT enhanced the damping properties significantly, especially at frequencies below $60 \mathrm{~Hz}$. The values of loss modulus were clearly higher (between $120 \%$ and $200 \%$, depending on the frequency level) than those of PBT-GF10 material, an indication of a higher energy dissipation potential of the material. A comparison of induced damage showed that TPEE composite had a stronger ability to initiate and accumulate damage and deform plastically. The Hysteresis loss at different strain rates and up to different stress levels in linear and nonlinear deformation regimes demonstrated TPEE enhanced the properties of storing and dissipating energy.

At the microscopic level, SEM observation showed that the interfacial fibre-matrix bonding was weakened by addition of TPEE and affected the deformation behaviour of the TPEE composite. Two types of debonding occurred in the TPEE matrix. One was observed as void stretching and micro-void formation around the fibres and the second was pull-out fibre-matrix deformation despite, relatively good adhesive behaviour (but not as efficient as for PBT-GF10). These local deformation zones at the interface around glass fibres affected the viscodamage behaviour in presence of TPEE and acted as dissipation zones, explaining the increase in the $\tan \delta$ value.

The weakness of interfacial adhesion in the TPEE composite caused loss of stiffness while the high local deformation during debonding enhanced the damping behaviour, with the energy-dissipation values calculated increased. It is believed that the damage and plastic deformation at microscopic level provided generally higher energy absorption of the TPEE composite resulting in higher damping and energy dissipation. 

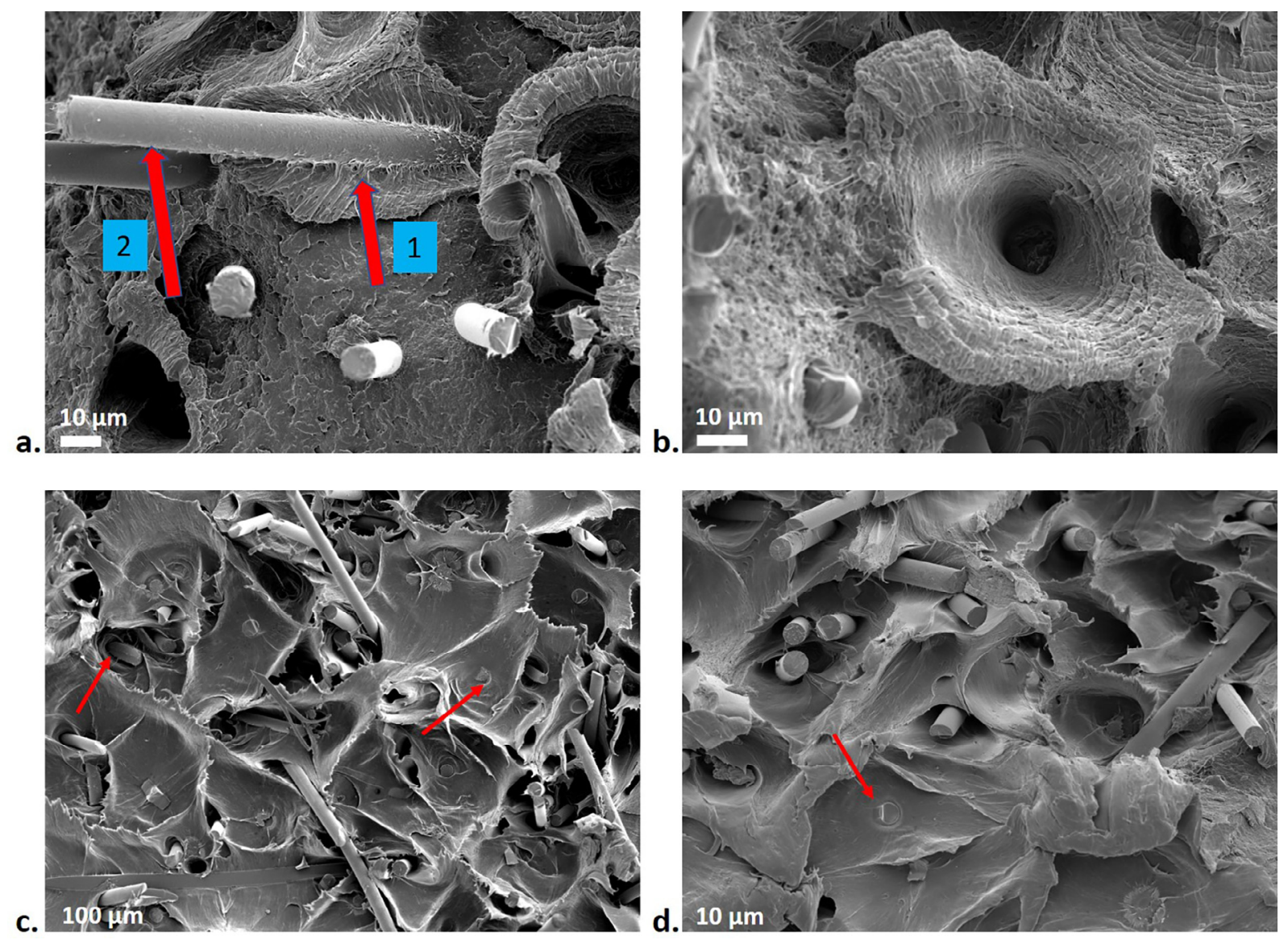

Fig. 13. Ductile areas with pulled-out fibres: (a) partially pulled-out fibre in PBT-GF10 TPEE; (b) conic structure as result of pulled-out fibre in material PBT-GF10 TPEE; (c) ductile area of PBT-GF10; (d) matrix strain around broken fibres due to pulling in PBT-GF10.

\section{Funding sources}

This research did not receive any specific grant from funding agencies in the public, commercial, or not-for-profit sectors.

\section{Declaration of Competing Interest}

The authors declare that they have no known competing financial interests or personal relationships that could have appeared to influence the work reported in this paper.

\section{References}

[1] Billmeyer FW. Textbook of polymer science. Wiley-Blackwell; 1984.

[2] Walker I, Collyer AA. Rubber toughening mechanisms in polymeric materials. In: Collyer AA, editor. Rubber toughened engineering plastics. Netherlands, Dordrecht: Springer; 2011. p. 29-56.

[3] Paul DR. Polymer blends vol. 1. Elsevier Science; 1978.

[4] McGrath GC. Fracture and toughening in fibre reinforced polymer composites. In: Collyer AA, editor. Rubber toughened engineering plastics. Netherlands, Dordrecht: Springer; 2011. p. 57-89.

[5] Keskkula H, Paul DR. Toughening agents for engineering polymers. In: Collyer AA, editor. Rubber toughened engineering plastics. Netherlands, Dordrecht: Springer; 2011. p. 136-64.

[6] Hourston DJ, Lane S, Zhang HX. Toughened thermoplastics: 3. Blends of poly(butylene terephthalate) with (butadiene-co-acrylonitrile) rubbers. Polymer (Guildf) 1995;36:3051-4.

[7] Aoyama T, Carlos AJ, Saito H, Inoue T, Niitsu Y. Strain recovery mechanism of PBT/ rubber thermoplastic elastomer. Polymer (Guildf) 1999;40:3657-63.

[8] Jha A, Bhowmick AK. Mechanical and dynamic mechanical thermal properties of heat- and oil-resistant thermoplastic elastomeric blends of poly(butylene terephthalate) and acrylate rubber. J Appl Polym Sci 2000;78:1001-8.

[9] Lim HCA. Thermoplastic polyesters. In: Gilbert M, editor. Brydson's plastic materials. eighth ed.Butterworth-Heinemann; 2016. p. 527-43.

[10] Jun JB, Park JG, Kim DH, Do Suh K. Blends of polybutyleneterephthalate with ethylene-propylene elastomer containing isocyanate functional group. Eur Polym J 2001;37:597-602.

[11] Aróstegui A, Gaztelumendi M, Nazábal J. Toughened poly(butylene terephthalate) by blending with a metallocenic poly(ethylene-octene) copolymer. Polymer (Guildf) 2001;42:9565-74.

[12] Archondouli PS, Kalfoglou NK. Compatibilization and properties of PBT/PU polymeric alloys. Polymer (Guildf) 2001;42:3489-502.

[13] Wang XH, Zhang HX, Wang ZG, Jiang BZ. Toughening of poly(butylene terephthalate) with epoxidized ethylene propylene diene rubber. Polymer (Guildf) 1997;38:1569-72.

[14] Gopalakrishnan J, Kutty SKN. Mechanical, thermal, and rheological properties of dynamically vulcanized natural rubber-toughened polystyrene. J Elastomers Plast 2015;47:153-69.

[15] Benson CM, Burford RP. Morphology and properties of acrylate styrene acrylonitrile/polybutylene terephthalate blends. J Mater Sci 1995;30:573-82.

[16] Viana JC. Polymeric materials for impact and energy dissipation. Plast Rubber Compos 2014;35:260-7.

[17] Verma G, Kulshreshtha B, Tyagi S, Ghosh AK. PBT/Thermoplastic elastomer blends - mechanical, Morphological, and rheological characterization. Polym Plast Technol Eng 2008;47:969-77.

[18] Wan C, Jiao Y, Sun Q, Li J. Agricultural, preparation, characterization, and antibacterial properties of silver nanoparticles embedded into cellulose aerogels. Polym Compos 2016;37:1137-42.

[19] Huang J, Wang J, Qiu Y, Wu D. Mechanical properties of thermoplastic polyester elastomer controlled by blending with poly(butylene terephthalate). Polym Test 2016;55:152-9.

[20] Kalfoglou NK. Thermomechanical studies of semicrystalline polyether-ester copolymers. Effect of thermal, mechanical, and solvent treatment. J Appl Polym Sci 1977;21:543-54.

[21] Yu Z, Wang X, Wu D. Mechanical properties, impact fracture behavior, and morphology of long-polyimide-fiber-reinforced poly(butylene terephthalate) composites. J Compos Mater 2017;51:3425-39.

[22] Ignaczak W, Wis̈niewska K, Janik J, El Fray M. Mechanical and thermal properties of PP/PBT blends compatibilized with triblock thermoplastic elastomer. Polish J Chem Technol 2015;17:78-83.

[23] Bai Y, Lv S, Liu F, Run MT. Preparation, morphology and properties of poly(trimethylene terephthalate)/thermoplastic polyester elastomer blends. J Macromol Sci Part B Phys 2014;53:1553-73.

[24] Shin H, Park ES. Mechanical and dielectric breakdown properties of PBT/TPE, PBT/ 
PBT/PET, and PBT/antioxidant blends. J Appl Polym Sci 2009;114:3008-15.

[25] Barkoula NM, Alcock B, Cabrera NO, Peijs T. Morphology, mechanical, and dielectric breakdown properties of PBT/PET/TPE, PBT/PET/PA66, PBT/PET/LMPE and PBT/PET/TiO2 blends. Polym Compos 2008;16:101-13.

[26] Qiu Y, Wu D, Xie W, Wang Z, Peng S. Thermoplastic polyester elastomer composites containing two types of filler particles with different dimensions: structure design and mechanical property control. Compos Struct 2018;197:21-7.

[27] Krenchel H. Fibre reinforcement: theoretical and practical investigations of the elasticity and strength of fibre-reinforced materials. Copenhagen: Akad. Forlag; 1964.

[28] Thomason JL. The influence of fibre length, diameter and concentration on the modulus of glass fibre reinforced polyamide 6,6. Compos Part A Appl Sci Manuf 2008;39:1732-8.

[29] Teixeira D, Giovanela M, Gonella LB, Crespo JS. Influence of injection molding on the flexural strength and surface quality of long glass fiber-reinforced polyamide 6.6 composites. Mater Des 2015;85:695-706.

[30] Libshitz HI, Banner MP. Understanding rheology of thermoplastic polymers. Radiology 1974;112:199-201.

[31] Zhang D, He M, Qin S, Yu J. Effect of fiber length and dispersion on properties of long glass fiber reinforced thermoplastic composites based on poly(butylene terephthalate). RSC Adv 2017;7:15439-54.

[32] Fitoussi J, Bocquet M, Meraghni F. Effect of the matrix behavior on the damage of ethylene-propylene glass fiber reinforced composite subjected to high strain rate tension. Compos Part B Eng 2013;45:1181-91.

[33] Sato N, Kurauchi T, Sato S, Kamigaito O. Microfailure behaviour of randomly dispersed short fibre reinforced thermoplastic composites obtained by direct SEM observation. J Mater Sci 1991;26:3891-8.

[34] Shonaike GO, Matsuo T. Fabrication and mechanical properties of glass fibre reinforced thermoplastic elastomer composite. Compos Struct 1995;32:445-51. 